\title{
A TABLE DRIVEN SEARCH APPROACH FOR REVELATION AND ANTICIPATION OF SINKHOLE ATTACK IN MANET
}

\author{
Girish Kumar Verma ${ }^{1}$, Rachit Jain ${ }^{2}$ \\ ${ }^{1}$ Research Scholar, Electronics \& Communication, ITM Universe, Gwalior, India, \\ girish2211@gmail.com \\ ${ }^{2}$ Assistant Professor, Electronics \& Communication, ITM Universe, Gwalior, India, \\ rachit.itm@gmail.com
}

\begin{abstract}
Sinkhole attack is likely one of the severe assaults in wireless ad hoc network. In sinkhole attack, compromised node or corrupt node broadcast unsuitable routing know-how to supply itself as a certain node and receives whole network visitors. After receiving whole community traffic it modifies the secret knowledge, comparable to alterations made to information packet or drops them to make the community difficult. A corrupt node affords to attract the safe data from all neighbouring nodes. In this thesis proposed a table driven search approach for revelation and anticipation of sinkhole attack in MANET in our proposed work first we initialize the route discovery process to communicate with destination node after this we store all the path information in node buffer who give path to destination now in node buffer we apply search process for find out common node in each path and mark node id after that send data to first given shortest path if attack is active than node wait for acknowledgement if ack is not arrived than mark node and send data to another node with common node id so that new path opt by neighbour by excluding this node id if attack is passive than we store the whole network knowledge so that we easily know neighbours of each node and then find out malicious node become easy task.
\end{abstract}

Keywords: MANET, Sinkhole.

\section{INTRODUCTION}

A Mobile Ad hoc Network demarcated as set of mobile nodes. This doesn't consist any fixed structure. As a result of it's less infrastructure network, the movable nodes within the network smartly set of communications with themselves to transmit packets from the idea to finish and this is often a self-organizing network. MANET has many applications like in military rescue operations piece of ground transmission, disaster unleash circumstances and industrial environments [1]. MANET have some security problems because of their inherent nature, like lack of centralized management, open medium, restricted battery power, dynamic topology and limited bandwidth Because of dynamically changing environment of MANET it's inclined for broad kind of attack [2].

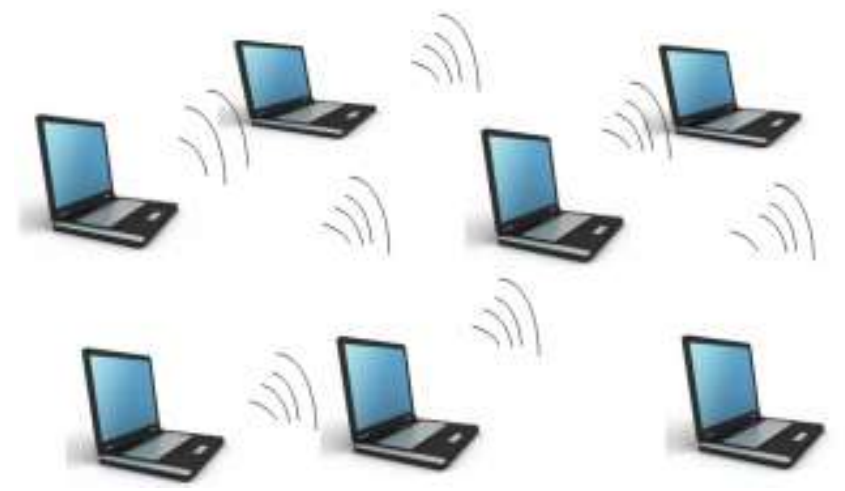

Fig-1: Mobile Ad-Hoc network.
In MANET there's not in the least a centralized administration. All nodes will execute as hosts and as routers. Also the nodes are mobile. So the topology changes frequently. MANETs permits the set of connections of networks right away during a fast manner, while not dependence on given facilities and infrastructure. During this manner, a corporation will discovered a network anyplace, either associated temporary (such as in response to a pressing scenario or in mobile armed operations) or during a permanent or semi-permanent manner (such as in border monitoring). This type of workability makes MANETs a useful addition to ancient networking technologies, most vital to a good deal of analysis and development. The Characteristics of MANET involves each disputes and possibilities in getting security objectives.

\section{Advantages of the Mobile ad-hoc network}

(i) They give data access regardless of the geographic situation.

(ii) Created at any time and place.

\section{SECURITY GOALS IN MANETS}

Security facilities contain functionality required to the present a secure networking atmosphere. The basic security facility can be summarized as follows: 


\subsection{Authentication:}

This facility authenticates identity of the use and assures the recipient that the information is from source that it claims to. Initially, communication initiation time, the facility assures that the authentic two different parties, that all entity is what it says. And next, it must assure that the third party doesn't interfere through impersonating one of the two authentic parties for the authorized transmission purpose and reception.

\subsection{Confidentiality:}

These facilities make sure that the knowledge transmitted over the network doesn't disclose to the unauthorized users. Confidentiality can be getting through applying various encryption methods.

\subsection{Access Control:}

This limits and also controls the resource access which can be an application or a host system.

\subsection{Integrity:}

Guarantee that the information is received in verbatim as sent through authorized users. The received information holds insertion, no modification or deletion.

\subsection{Anonymity:}

Means of the anonymity that all knowledge that can used to vendor recognizes or the existing user of node should contain private default and not be dispersed through the node itself or software system. This measure is closely privacy preserving related, in which we should try to protect node privacy from arbitrary disclosure to any several entities.

\subsection{Non Repudiation:}

Non repudiation guarantees that the receiver and the sender of information cannot be disavow that they have ever received or sent such information. This is valuable particularly when we need to the discriminate if any node with a number of abnormal performance is compromised or not if a node recognize that the information it has find is erroneous, it can then use the improper introduction as an evidence to inform other different nodes that node sending out of the faulty knowledge should have been cooperate[3].

\section{SECURITY ATTACKS IN MANET}

On the basis of manners of outbreaks safety outbreaks in MANET can be classified on the basis of the foundation of the attacks i.e. External or Internal, and on the activities of the attack i.e. Active or Passive attack. This classification is important because the network can be misused by attackers both as external, internal with passive or active attack against the network.

\subsection{External and Internal Attack}

External attackers are present remote systems who need to turn into familiar with the system and formerly they turn into familiar with the system they start transfer fake bundles, rejection of direction consequently as to disturb the implementation of the complete system. This attack is similar to the attacks that are made in opposition to wired system. These attacks can be preventing by efforts to set up security, such as, firewall, where the access of unapproved entity to the system can be modest. While in inner attack the aggressor requirements to have distinctive access to the system and additionally take part in the usual movements in system. The attacker finds access of the system because original node either by trading off a current node in the system or by harmful mimics and starts its malicious ways. Inner attack is more precise attacks then outer attacks.

\subsection{Passive Attacks:}

Such assault cannot be change the information sending in the network. For the network traffic it involves the unauthorized "listening" [15]. This kind of assailant does not interfere the procedure of a routing protocol except tries to discover the necessary information from routed traffic. Eavesdropping, traffic analysis, and Monitoring are some attacks.

\subsection{Active Attacks:}

These attacks are hazardous attacks that discontinue messages overflow among the nodes. Active attacks can be external or internal. Internal attacks are from suspicious nodes which are network part. These attacks are very harm and dangerous to observe than external attacks. External attacks can be accepted by exterior sources that do not fit in to the network. Unauthorized access to network generated by these attacks that support the assailant to create alteration for example alteration of packets. Some active attacks are Spoofing, Wormhole, Fabrication attack, Sybil attack, Denial of services attack and Sinkhole attack, [15].

\section{CHALlangeS IN DETECTING SINKHOLE}

\section{ATTACK}

In lightweight of our review of the literature on sink attacks in MANETs, the concomitant area unit the basic difficulties in recognizing sinkhole attack:

\subsection{Specific Sinkhole Attacks Depend On Routing Protocols:}

In MANETs packets are transmitted in light of a routing metric which shifts for various routing protocols. For instance the procedures utilized by a traded off node in network that uses the Tiny AODV protocol will be not the same as the one utilized another convention, for example, the MintRoute protocol. Be that as it may, these protocols are for the most part in light of how "close" a node is to the base station. An attacking node can abuse this to deceive its neighbors keeping in mind the end goal to dispatch a 
sinkhole attack. At that time all the information from its neighbors to the base station can undergo the assailant node.

\subsection{Sinkhole Attacks May Be Insider Attacks:}

Insider and outsider attacks are two classes of attacks on networks. A outsider attack adds a malicious node to the network. In an insider attack the interloper bargains one of the legitimate nodes by messing with it or through shortcoming in the victim's system software; compromised nodes infuse false data in the network or listen to secret data. A compromised node has satisfactory access benefit in the system and right now has learning relating to the system topology which makes extra difficulties in location. Because of this circumstance, even cryptography may not by any means shield against insider attack. Thusly insider attacks represent a greater number of genuine danger to frameworks than outsider attacks.

\subsection{Resource Constraints Limit Detection Methods:}

The restricted power provide, low correspondence vary, low memory limit and low process force of nodes square measure the elemental necessities in MANETs that hinder usage of solid security systems. The solid scientific discipline techniques utilised as a neighborhood of various networks cannot be dead in an exceedingly MANET because of low process power and low memory limit. During this manner weaker system excellent with accessible assets should be utilised.

\section{LITRATURE SURVEY}

Manisha(2013 ) et al [15] present that network platforms are less costly and more influential incorporating small electronic devices named as Motes. Networks improve its reputation in defense and health centric research region; as well as accepted in industrial region. Author presents the security perquisites as MANETs are simply vulnerable to more attacks than wired networks.

Rajkumar (2013) et al [16], in respect to give whole resolution to identify and avoid sinkhole attack a Leader Based Intrusion Detection System is devised. In this approach a leader is chosen for every group nodes within the network, region wise and it equates and estimates the nature of each node sensibly performs detection module and observes every node nature among the group for any sinkhole attack to take place. When a node gets recognized as a affected node, it notify that nodes status to the other leader within the WSN, such that every leaders present in the network are aware of the sink hole node and the leaders discontinue transmission with sinkhole node. In this technique they enhanced the performance of the system by means of energy efficiency and intrusion detection rate.

Tomar (2014) et al [17] Grants mechanism of isolation and detection of sinkhole outbreak in MANET, replacement of routing protocol to enhance network ability after grievous attack from AODV routing protocol. The suggested protocol has five phases. Initialization phase, table \&storage maintain phase, route inquiry phase, route resumption phase and assault analysis. The primary phase is initialization phase, source node shocks route discovery through broadcasting request packet to every neighbour Nodes to gain shortest, robust in positions of long life or energy efficient. 2nd phase is, routing table modernize\& storing sequence number, hop count and node id of Source/destination and many others within the routing table of nodes. $3^{\text {rd }}$ phase, route inquiry phase to evaluate sequence number of previous and current request. Fourth phase is, Assault analysis, now we have assumed that a threshold price of sequence number. In the end last segment is route resumption phase. On this phase, we can analyze sequence number and threshold value compare with that are close values then make effort to establish numerous methods between source nodes to destination node to forestall on sinkhole attack.

\section{PROBLEM STATEMENT[17]}

1. The existing algorithm fails when the number of malicious node increases.

2. Fails if path from source to destination is always through malicious node.

3. Algorithm only detects but does not prevent the sinkhole attack.

4. Detection of active attack is not possible.

\section{PROPOSED MATHODOTOLOGY}

MANET is constructing the new era for the next-generation wireless world. Where packet delivers happen in infrastructure less scenario, or fault or malicious behaviour detection find out is very difficult. Mobility or distributed architecture has the major issue to identify malicious behaviour in this network. Malicious behaviour of nodes reflected by moving condition in existing condition malicious behaviour detect or isolate with the help of shortest path or select multipath for forward packet but all the time this method does not work overcome these problem we proposed a table driven search approach for revelation and anticipation of sinkhole attack in MANET in our proposed work first we initialize the route discovery process to communicate with destination node after this we store all the path information in node buffer who give path to destination now in node buffer we apply search process for find out common node in each path and mark node id after that send data to first given shortest path if attack is active than node wait for acknowledgement if ack is not arrived than mark node and send data to another node with common node id so that new path opt by neighbour by excluding this node id if attack is passive than we store the whole network knowledge so that we easily know neighbours of each node and then find out malicious node become easy task.

Algorithm.

Step1: initialize ();

Step2: store path info ();

Step3: for $(\mathrm{i}=0 ; \mathrm{i}<$ tablepath.length; $\mathrm{i}++)\{$

If $($ nodeid $[\mathrm{i}]==$ nodeid $[\mathrm{i}++])\{$

Mark common node; \} 
Step4: initialize $\mathrm{t}=0$;

Step5: if (ACK!) \{

$\mathrm{t}++$;

if $(\mathrm{t}>=2)\{$

mark node as a malicious; $\}$

Else \{

Send data to this path

Step6: initialize network known process ();

Step7: if (destination neighbor does not match ) \{

Mark node as a malicious node; $\}$

Step8: block node;

Step9: exit;

\section{RESULT \& SIMULATION}

The simulation is done on NS-2 with maximum number of nodes 20 , queue length 50 , xy dimension is $1100 \times 1100$ the start and end of simulation are 1.0 and $100.0 \mathrm{~ms}$ respectively. Routing protocol used is AODV and type of antenna is omni-directional. The below given table. 1 depicts the simulation parameters and their respective values.

Table-1: Simulation Parameters

\begin{tabular}{|l|l|}
\hline Simulation Parameters & values \\
\hline Number of nodes & 20 \\
\hline Routing protocol & AODV \\
\hline XY- dimension & $1100 \mathrm{X} 1100$ \\
\hline Queue length & 50 \\
\hline antenna & Omni-directional \\
\hline Start simulation & $1.0 \mathrm{~ms}$ \\
\hline End simulation & $100.0 \mathrm{~ms}$ \\
\hline
\end{tabular}

\section{Node Energy:}

The amount of energy required by a node for processing the data that has been received. By node energy we mean that, a node is able to conserve its energy in an efficient manner. Node energy utilization is an severe issue in MANET.

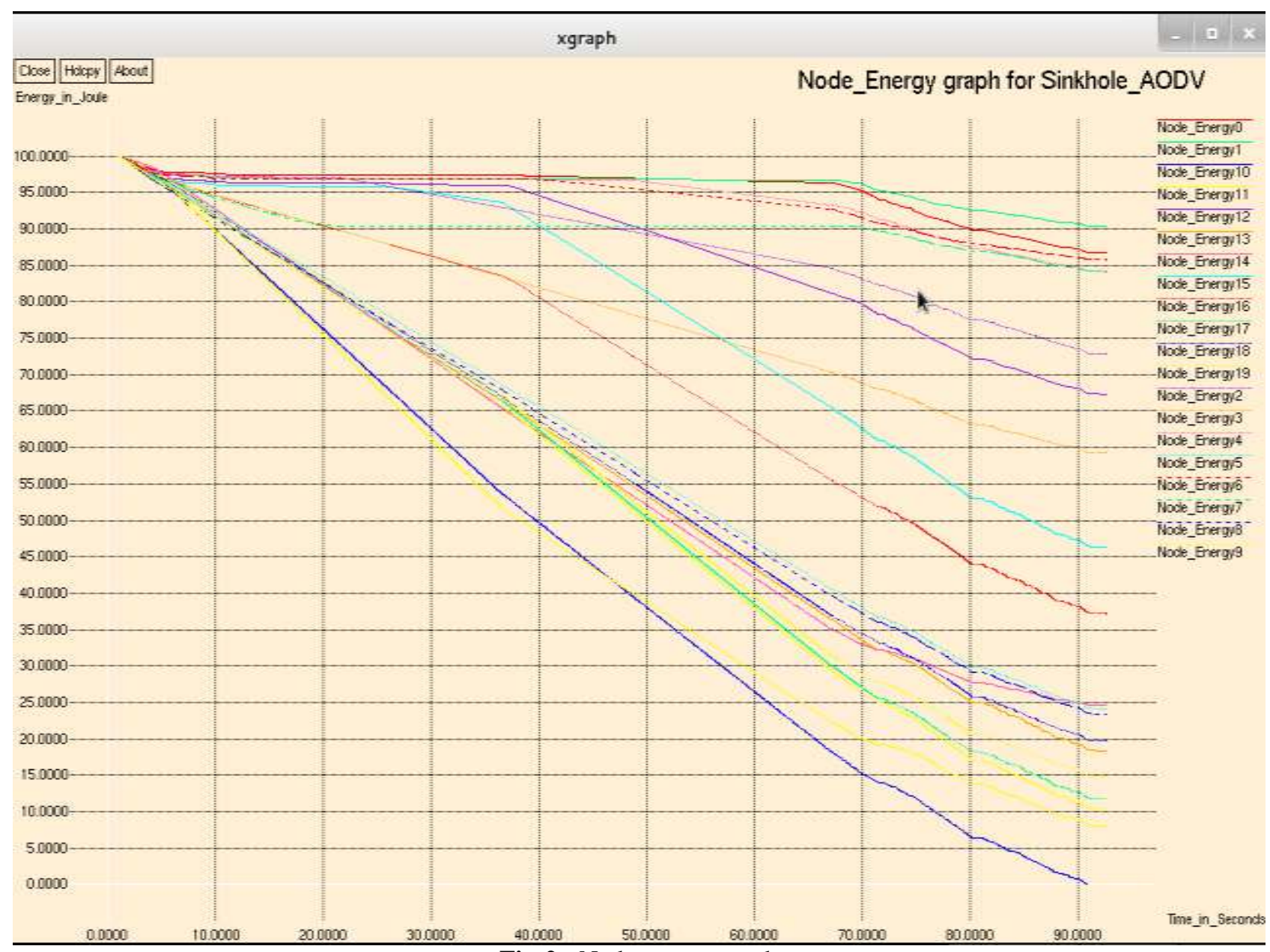

Fig-2: Node energy graph

\section{Packet delivery ratio:}

It is defined as the ratio of packets delivered to the destinations that generated by the sender mathematically, termed as:
$\mathrm{PDR}=\mathrm{R} 1 \div \mathrm{R} 2$

Where, R1 is the total number of packets arrived at every destination and $\mathrm{R} 2$ is the total number of packets created by the every source. 


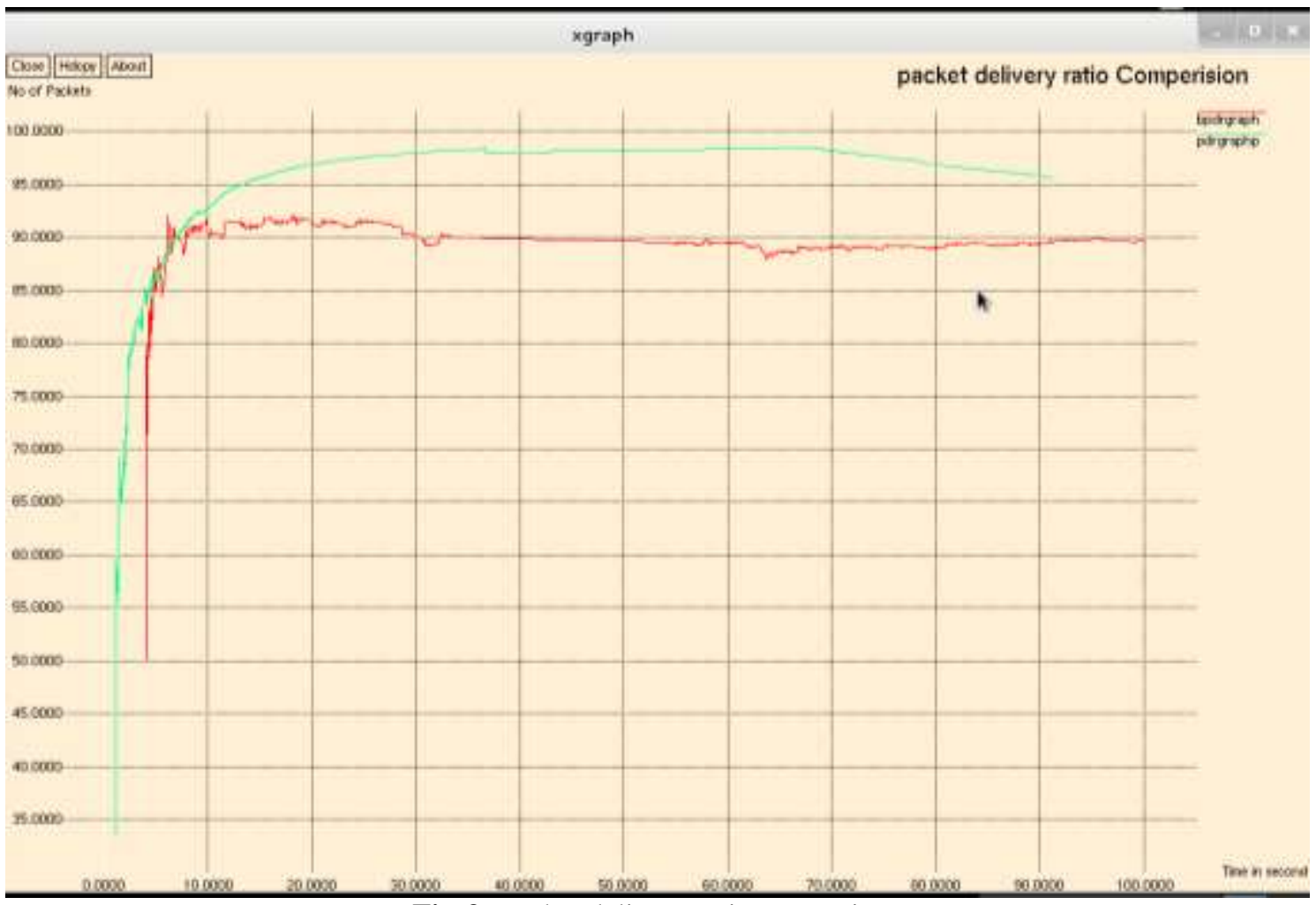

Fig-3: Packet delivery ratio comparison

\section{Throughput:}

It is refers to as amount of packets delivered above the total simulation time. Equating the throughput demonstrate that the three algorithms performance limitations are extremely near load of traffic 50 and 100 nodes in MANET scenario.
Throughput $=\mathrm{N} / 1000$

here $\mathrm{N}$ is the number of bits arrived at the recipient successfully

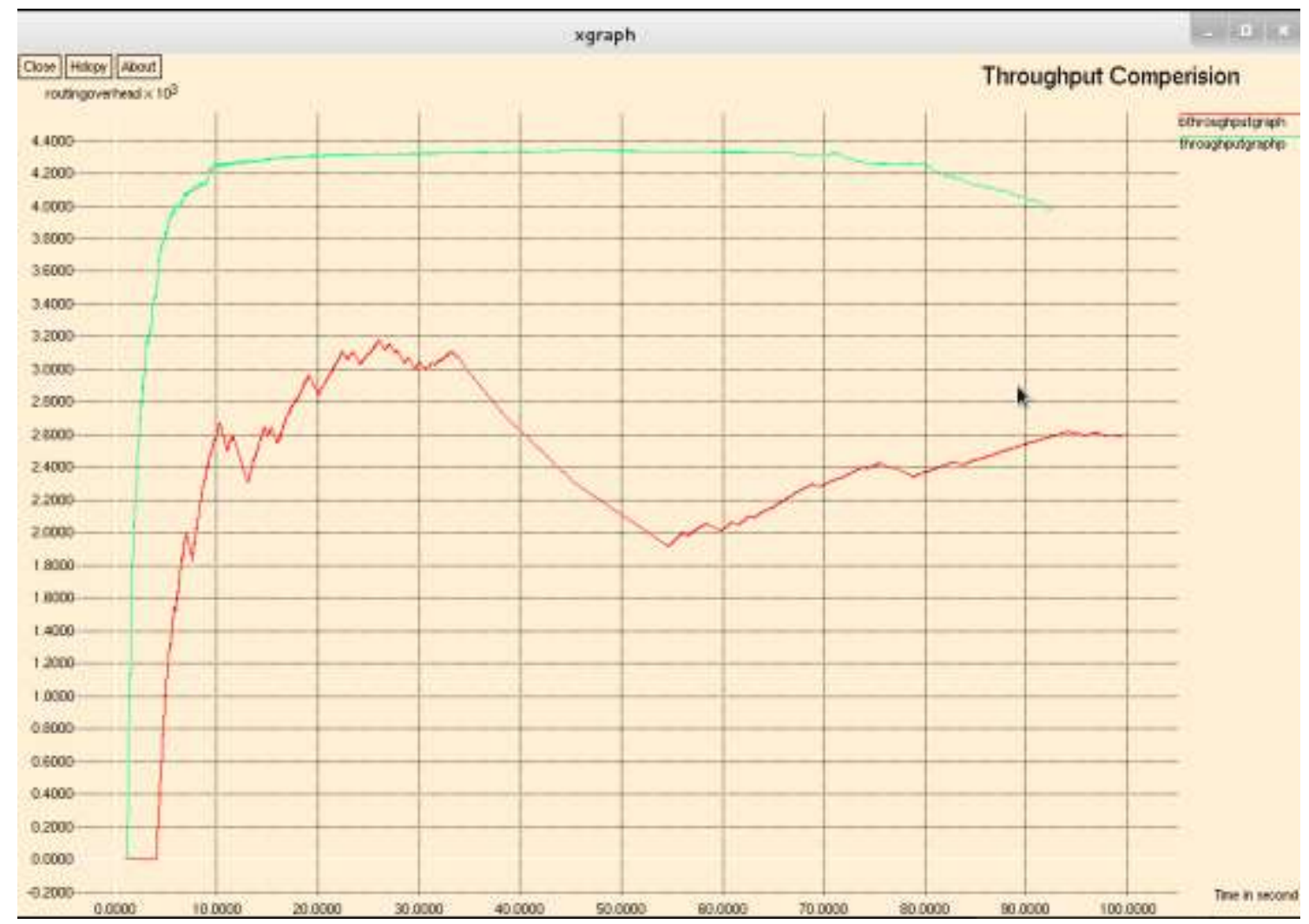

Fig-4: Throughput Comparison graph 


\section{CONCLUSION}

MANET is constructing the new era for the next-generation wireless world. Where packet delivers happen in infrastructure less scenario, or fault or malicious behavior detection find out is very difficult. Mobility or distributed architecture has the major issue to identify malicious behavior in this network. Malicious behavior of nodes reflected by moving condition in existing condition malicious behavior detect or isolate with the help of shortest path or select multipath for forward packet but all the time this method does not work overcome these problem we proposed A Table Driven Search Approach for Revelation and Anticipation of Sinkhole Attack In MANET.

The proposed approach posses good results in terms of node energy, packet ratio and throughput. In future, can apply hidden markov model for that data sets needs to be trained, the trained data will follow the property of honest node. This data set will be able to detect the malicious nodes behavior.

\section{REFERENCES}

[1] Nitesh A. Funde1, P. R. Pardhi "Detection \& Prevention Techniques to Black \& Gray Hole Attacks In MANET: A Survey International Journal of Advanced Research in Computer and Communication Engineering Vol. 2, Issue 10, October 2013

[2] V. Shanmuganathan Mr.T.Anand M.E.," A Survey on Gray Hole Attack in MANET" IRACST - International Journal of Computer Networks and Wireless Communications (IJCNWC), ISSN: 2250-3501 Vol.2, No6, De cember 2012

[3] Meenakshi Yadav , Nisha Uparosiya "Survey on MANET: Routing Protocols, Advantages, Problems and Security" International Journal of Innovative Computer Science \& Engineering Volume 1 Issue 2; Page No. 1217

[4] Deepak Chayal and Dr. Vijay Singh Rathore "Assessment of Security in mobile Ad-hoc Network (MANET)" Journal of Global Research in Computer Science Volume 2, No. 6, June 2011

[5] Pooja Jaiswal and Dr. Rakesh Kumar "Prevention of Black Hole Attack in MANET" IRACST International Journal of Computer Networks and Wireless Communications (IJCNWC), ISSN: 22503501 Vol.2, No5, October 2012

[6] M.izaz, "Transmission control protocol (TCP) Performance Evaluation in MANET" Mar2009

[7] Ahmad Salehi S., M.A. Razzaque, Parisa Naraei, Ali Farrokhtala;" Detection of Sinkhole Attack in Wireless Sensor Networks". Proceeding of the 2013 IEEE International Conference on Space Science and Communication (IconSpace), 1-3 July 2013

[8] S. D. Roy, S. A. Singh, S. Choudhury and N. C. Debnath, "Countering sinkhole and black hole attacks on sensor networks using Dynamic Trust Management", 2008 IEEE Symposium on Computers and Communications, (2008), pp. 537-542.
[9] J. Sen, "A Survey on Wireless Sensor Network Security”, Int. J. Commun. Networks, vol. 1, no. 2, (2009), pp. 59-82.

[10] Chen C, Song M, Hsieh G. Intrusion detection of sinkhole attacks in large-scale wireless sensor networks. In: Proceedings of wireless communications, networking and information security (WCNIS). IEEE; 2010. p. 711e6.

[11]P. Samundiswary PP, Dananjayan P. Detection of sinkhole attacks for mobile nodes in heterogeneous sensor networks with mobile sinks. International Journal of Computer and Electrical Engineering 2010;2:127e33.

[12] Sharmila S, Umamaheswari G. Detection of sinkhole attack in wireless sensor networks using message digest algorithms. In:Proceedings of process automation, control and computing (PACC), IEEE. IEEE; 2011. p. $1 \mathrm{e} 6$.

[13]D Sheela Nk, Mahadevan G. A non cryptographic method of sinkhole attack detection in wireless sensor networks. In:Proceedings of IEEE-international conference on recent trends in information technology 2011. p. $527 \mathrm{e} 32$.

[14] Maliheh Bahekmat, Mohammad Hossein Yaghmaee, Ashraf Sadat Heydari Yazdi, and Sanaz Sadeghi;" A Novel Algorithm for Detecting Sinkhole Attacks in WSNs".International Journal of Computer Theory and Engineering, Vol. 4, No. 3, June 2012

[15] Manisha, Gaurav Gupta," Attacks on Wireless Sensor Networks: A Survey",International Journal of Advanced Research in Computer Science and Software Engineering,Volume 3, Issue 10, October 2013, Page 190- 197

[16]Udaya Suriya Rajkumar, D. and Rajamani Vayanaperumal;" A Leader Based Monitoring Approach For Sinkhole Attack In Wireless Sensor Network". International Journal of Computer Science, 2013.

[17] Shashi Pratap Singh Tomar,Brijesh Kumar Chaurasia;" Detection and Isolation of Sinkhole Attack from AODV Routing Protocol in MANET". Sixth International Conference on Computational Intelligence and Communication Networks, 2014 